CARDIOVASCULAR DISORDERS

\title{
Resolving blood clots
}

Current therapies for deep vein thrombosis (DVT) - which can occur as a result of immobilization, surgery and pregnancy — such as heparins, warfarin and the newgeneration anticoagulants act on the coagulation cascade rather than directly targeting the thrombus. Now, Nosaka and colleagues show that inhibiting interferon- $\gamma$ (IFN $\gamma)$ can accelerate thrombus resolution in a mouse model of DVT.

As the processes that are involved in thrombus resolution resemble those that are involved in wound healing, in which the IFN $\gamma$-signal transducer and activator of transcription 1 signalling pathway is involved, the authors examined the role of IFN $\gamma$ in thrombus resolution in IFN $\gamma$ deficient $\left(\right.$ Ifng $\left.{ }^{-/}\right)$mice and in a mouse model of DVT induced by ligation of the inferior vena cava (IVC).

In the mouse model, IFN $\gamma$ was present in macrophages that infiltrated the thrombus, and its expression increased as thrombi aged. In Ifng ${ }^{-/-}$mice, thrombi started to reduce in size 10 days after IVC ligation and were smaller in size and mass than the thrombi in wild-type mice, which suggested that the absence of IFN $\gamma$ could accelerate thrombus resolution.

Matrix metalloproteinase 2 (MMP2) and MMP9 have crucial roles during thrombus resolution, and work suggested that IFN $\gamma$ suppresses the expression and activity of MMP9 in macrophages that are present in the thrombus. Intrathrombotic recanalization the formation of new vascular channels within the thrombus - is also necessary for thrombus resolution, and Ifng $\mathrm{g}^{--}$mice had increased numbers of intrathrombotic channels and improved blood flow through the IVC region. Furthermore, intrathrombotic expression of vascular endothelial growth factor (Vegf) was enhanced in Ifng ${ }^{-/-}$mice. In vitro studies confirmed that IFN $\gamma$ negatively regulated the expression of $M m p 9$ and Vegf in macrophages.

Finally, administration of a monoclonal antibody $(\mathrm{mAb})$ directed against IFN $\gamma$ to mice resulted in reduced thrombus mass and increased blood flow through the thrombus, which were accompanied by increased expression of $M m p 9$ and Vegf. Moreover, the mAb did not cause any abnormalities in coagulation function. So, this study indicates that inhibiting IFN $\gamma$ could be beneficial in the treatment of DVT and possibly other thrombotic disorders.

Charlotte Harrison

ORIGINAL RESEARCH PAPER Nosaka, M. et al. Absence of IFN- $\gamma$ accelerates thrombus resolution through enhanced MMP-9 and VEGF expression in mice.J. Clin. Invest. 121, 2911-2920 (2011)

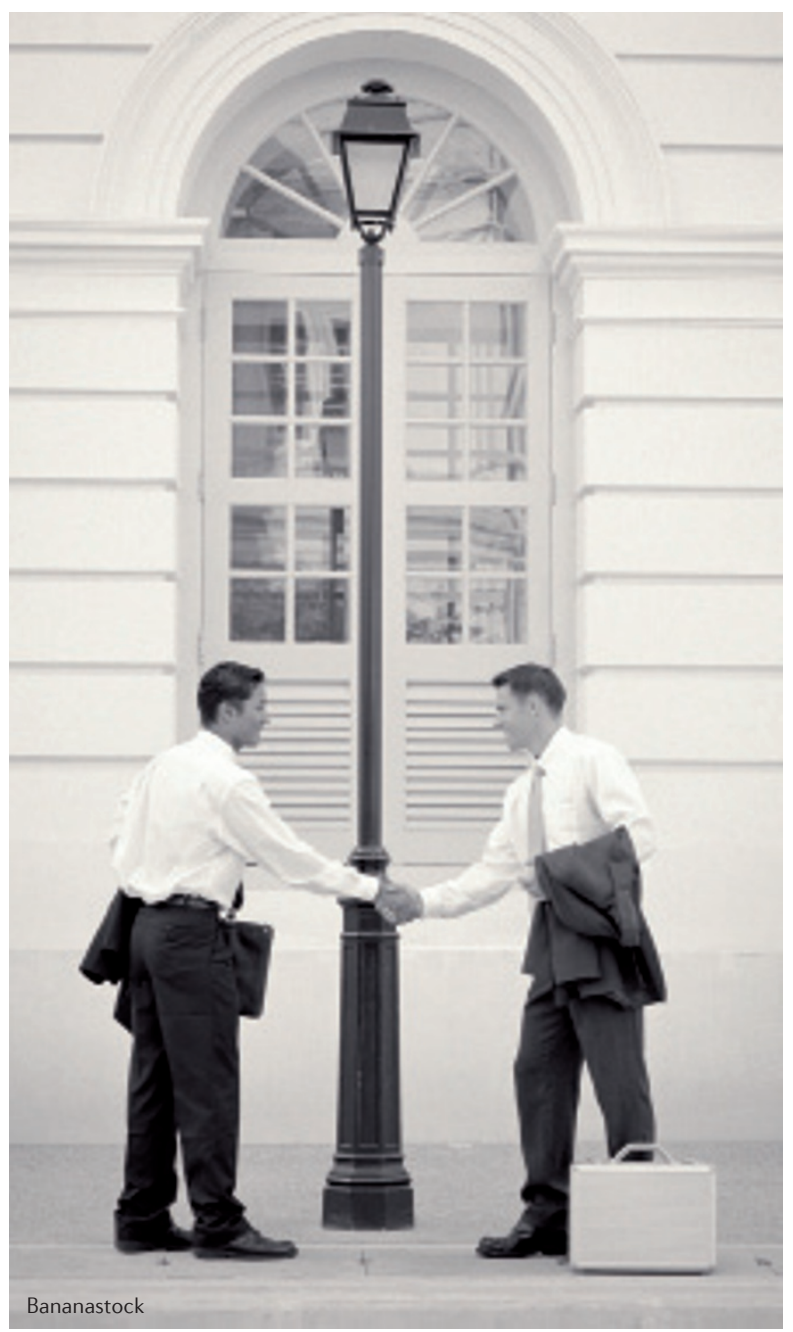

